\title{
HBV Pre-S1-Derived Myristoylated Peptide (Myr47): Identifi- cation of the Inhibitory Activity on the Cellular Uptake of Lipid Nanoparticles
}

\author{
Masaya Nanahara ${ }^{1,2,3}$, Ya-Ting Chang ${ }^{1,4}$ Masaharu Somiya ${ }^{1}$ and Shun'ichi Kuroda ${ }^{1,2, *}$ \\ 1 The Institute of Scientific and Industrial Research (ISIR-Sanken), Osaka University, Osaka 567-0047, Japan; \\ skuroda@sanken.osaka-u.ac.jp \\ 2 Graduated School of Frontier Biosciences, Osaka University, Osaka 565-0871, Japan; \\ msomiya44@sanken.osaka-u.ac.jp \\ 3 Advanced Photonics and Biosensing Open Innovation Laboratory (PhotoBIO-OIL), AIST-Osaka University, \\ Osaka 565-0871, Japan; nanahara44@sanken.osaka-u.ac.jp \\ 4 College of Life Science, National Taiwan University, Taipei 10617, Taiwan; blair8510@gmail.com \\ * Correspondence: skuroda@sanken.osaka-u.ac.jp; Tel.: +81-6-6879-8460
}

\begin{abstract}
Myr47 lipopeptide consisting of hepatitis B virus (HBV) pre-S1 domain (myristoylated 2-48 peptide) is a commercialized effective anti-HBV drug, preventing the interaction of HBV with sodium taurocholate cotransporting polypeptide (NTCP) on human hepatocytes, of which the activity requires both $\mathrm{N}$-myristoylation residue and specific amino acid sequence. Meanwhile, we recently reported that Myr47 reduces the cellular uptake of HBV surface antigen (HBsAg, subviral particle of HBV) in the absence of NTCP expression (Somiya; et al. Virology 2016, 497, 23-32). In this study, we analyzed how Myr47 reduces the cellular uptake of lipid nanoparticles (including liposomes (LPs) and HBsAg) without NTCP expression. By using Myr47 mutants lacking the HBV infection inhibitory activity, they could reduce the cellular uptake of LPs in an $\mathrm{N}$-myristoylation-dependent manner whereas in an amino acid sequence-independent manner. Moreover, Myr47 and its mutants could reduce the interaction of LPs with apolipoprotein E3 (ApoE3) in an N-myristoylation-dependent manner regardless of their amino acid sequences. From these results, N-myristoyl residue of lipopeptides generally could interfere the LPs/HBsAg-ApoE3 complex formation, thereby reducing the cellular uptake of LPs/HBsAg. When lipid nanoparticles are used as a DDS (drug delivery system) nanocarrier, the surface modification with lipopeptides may be a new method to inhibit unwanted cellular uptake of DDS nanocarriers by non-target cells.
\end{abstract}

Keywords: hepatitis B virus (HBV); Myr47 lipopeptide; cellular uptake; liposomes; sodium taurocholate cotransporting polypeptide (NTCP); HBV surface antigen (HBsAg); apolipoprotein E (ApoE)

\section{Introduction}

Hepatitis B virus (HBV) is a DNA virus specifically infecting human liver hepatocyte, and causes serious liver diseases such as hepatitis, cirrhosis, and liver cancer. Although HBV was discovered nearly half century ago, the curative agent has not been developed so far [1], while only $\mathrm{HB}$ vaccine could prevent the horizontal and vertical transmission of HBV. HBV is an enveloped virus containing three types of envelope proteins called S, M, and L protein that share the same C-terminal sequence [2]. Especially, the pre-S1 region of L protein is essential for specific recognition of human hepatocytes [3-5]. N-terminal 3-77 amino acid residues (aa) of pre-S1 region [6] and myristoyl residue at $\mathrm{N}$-terminus of pre-S1 region $[7,8]$ are indispensable for cell entry process. Recently, an essential region for hepatocyte recognition was identified as 9-18 aa of pre-S1 region [9]. In 2012, sodium taurocholate cotransporting polypeptide (NTCP) 
that is specifically expressed in hepatocyte, was identified as a critical cellular receptor for HBV early infection (cell entry step) [10].

As a promising agent for the prevention of HBV infection, pre-S1-derived peptide encompassing 2-48 aa with N-myristoyl residue (hereafter Myr47) can inhibit the cellular entry of HBV by repressing the interaction between NTCP and HBV in competitive manner [11]. Currently, Myr47 and its derivatives (Hepcludex, Bulevirtide, Myrcludex-B) are on the market in EU countries for the treatment of chronic HDV patients with HBV superinfection.

Meanwhile, our group reported that Myr47 inhibits the cellular uptake of HBsAg (HBV surface antigen, subviral particle of HBV) by human hepatocellular carcinoma-derived HepG2 cells (no NTCP expression) [11]. In this study, we examined if Myr47 inhibits the cellular uptake of lipid nanoparticles (liposomes (LPs) and HBsAg) by HepG2 cells in the absence of NTCP. We investigated the effect of N-myristoyl residue and the amino acid sequence of Myr47 on the inhibition of cellular uptake. In addition, since ApoE3 (human apolipoprotein E3) was shown to play an important role in the cellular uptake of HBV [12] and the elimination of LPs from blood by hepatocytes [13], we found N-myristoyl residue of Myr47 inhibits the formation of the LPs-ApoE3 complex.

\section{Materials and Methods}

\subsection{Materials}

Biotinylated Myr47 lipopeptide was synthesized by Scrum. Biotinylated mutated Myr47 lipopeptides (aa2-48, D-11,13, Ala11-15, Scrambled (Scr)[14]) were purchased from Biologica. DOPC (1,2-Dioleoyl-sn-glycero-3-phosphocholine) was purchased from NOF. Cholesterol and CellVue Claret were purchased from Sigma-Aldrich. DiD (1,1'-dioctadecyl-3,3,3',3'-tetramethylindodicarbocyanine, 4-chlorobenzenesulfonate salt) was purchased from Thermo Fisher Scientific. Anti-FITC IgG and anti-human apolipoprotein E3 IgG were purchased from GeneTex. 18:1 FITC cap PE (1,2-dioleoyl-sn-glycero-3-phosphoethanolamine-N-(carboxyfluorescein) (ammonium salt)) was purchased from Avanti Polar Lipids. Alphascreen Protein A detection kit was purchased from PerkinElmer Japan. Human apolipoprotein E3 (ApoE3) was purchased from Peprotech. Hepatitis B patient's plasma-derived HBsAg particles and mouse monoclonal anti-S IgG (clone824) were purchased from Institute of Immunology.

\subsection{Cell Culture}

Human hepatocellular carcinoma HepG2 cells (JCRB Cell bank) were cultured in DMEM (Dulbecco's modified Eagle medium) containing 10\% FBS (fetal bovine serum), $100 \mathrm{U} / \mathrm{mL}$ penicillin, and $100 \mu \mathrm{g} / \mathrm{mL}$ streptomycin at $37^{\circ} \mathrm{C}$ in $5 \% \mathrm{CO} 2$ humidified incubator.

\subsection{Liposomes (LPS)}

Each of DOPC, cholesterol, DiD, and 18:1 FITC cap PE was dissolved in ethanol as 1 $\mathrm{mg} / \mathrm{mL}$. Lipids solutions were mixed (DOPC: cholesterol $=6: 4(\mathrm{~mol} / \mathrm{mol})$ with/without 0.1 $\mathrm{mol} \%$ of DiD or $2 \mathrm{~mol} \%$ of 18:1 FITC cap PE), and injected to the 9-fold volume of PBS (phosphate-buffered saline) with vortexing. Subsequently, the mixture was sonicated by Branson sonifier 450 at RT (room temperature) for 20 seconds and used immediately. The diameter and zeta potentials of LPs were measured by dynamic light scattering method using Nanosizer (Malvern). LPs used in this study are approximately $61 \mathrm{~nm}$ in diameter, PDI (particle diversity index $)=0.195$, and $-0.649 \mathrm{mV}$ zeta potentials.

\subsection{Confocal Laser Microscopy}

HepG2 cells $\left(2 \times 10^{5}\right.$ cells/well $)$ were inoculated in 8 -well glass bottom chamber one day before experiment. Immediately after Myr47 (500 nM) injection, DiD-labeled LPs 
$(10 \mu \mathrm{g} / \mathrm{mL})$ were added to the wells. After 3 hours incubation, the cells were washed twice with PBS, and fixed by $4 \%(\mathrm{w} / \mathrm{v})$ paraformaldehyde in PBS $(400 \mu \mathrm{L} /$ well $)$ containing Hoechst $33342(2 \mu \mathrm{g} / \mathrm{mL})$ for 20 minutes. The cells were subsequently washed once with PBS, and the Fluoro-KEEPER Antifade Reagent (nacalai tesque) was added to the wells. The cells were observed under confocal laser microscopy FV1000 (Olympus).

\subsection{Flow Cytometry}

HepG2 cells $\left(1.5 \times 10^{5}\right.$ cells/well) were inoculated in 12-well plate one day before experiment. Medium was changed to DMEM without FBS. Immediately after ApoE3 (25 $\mu \mathrm{g} / \mathrm{mL}$ ) injection, Myr47 (500 nM) was added to the medium, and then DiD-labeled LPs $(5 \mu \mathrm{g} / \mathrm{mL}$ ) or CellVue-labeled HBsAg ( $5 \mu \mathrm{g} / \mathrm{mL}$ as protein) were added to the medium. After 3 hours incubation, the medium was removed, and the cells were detached with 5 mM EDTA in PBS and subjected to Flow cytometer FACS Canto II (BD Bioscience). Data were analyzed by FlowJo7.6.5 program. For labeling HBsAg with CellVue Claret, HBsAg $(500 \mu \mathrm{g} / \mathrm{mL}$ as protein) in PBS was incubated with the same volume of CellVue solution (250-fold dilution) at RT for 5 minutes and mixed with 4-fold volume of DMEM containing $10 \%$ FBS to stop labeling reaction.

\subsection{Alphascreen ${ }^{\circledR}$ Assay}

Alphascreen Protein A detection kit was used with Streptavidin beads. Mixture of 1 $\mathrm{M} \mathrm{NaCl}, 1 \mathrm{M}$ Tris- $\mathrm{HCl}(\mathrm{pH}=8.0)$, and $1 \%(\mathrm{w} / \mathrm{v}) \mathrm{BSA}$ (bovine serum albumin) was used as sample buffer. FITC-LPs (300 ng) and either Myr47 or its mutants (50 ng) in $15 \mu \mathrm{L}$ sample buffer were incubated in 384-well microplate at RT for 30 minutes. Subsequently, for fixation of LPs on Protein A beads, anti-FITC IgG (2 ng) in $5 \mu \mathrm{L}$ sample buffer was injected to each well. Then, the plate was incubated at RT for 30 minutes. After that, Protein A beads and Streptavidin beads ( $0.1 \mu \mathrm{L}$ each) in $10 \mu \mathrm{L}$ sample buffer were added to each well, and the plate was incubated at RT for 1 hour. The interaction between LPs and Myr47 or its mutants was quantified by measuring the fluorescence intensity. As for the interaction between HBsAg and Myr47 or its mutants, HBsAg (50 ng as protein) and anti-S domain IgG (2 ng) were used instead of FITC-LPs and anti-FITC IgG, respectively.

\subsection{Bio-Layer-Interferometry (BLI) Analysis}

Unless otherwise described, all operations were performed with BLItz (Fortebio) at RT with agitation at 2,200 rpm. Anti-Human ApoE3 IgG $(1 \mu \mathrm{M})$ was contacted with Protein A sensor chip (Fortebio) for 240 seconds. The sensor chip was washed with K-buffer (PBS containing 0.1\% BSA) for 30 seconds. ApoE3 $(2.5 \mu \mathrm{M})$ could bind with antibody on the sensor chip for 120 seconds. The sensor chip was washed with K-buffer for 120 seconds. Meanwhile, mixture of LPs $(500 \mu \mathrm{g} / \mathrm{mL})$ and Myr47 or its mutants (50 $\mu \mathrm{g} / \mathrm{mL}$ ) was incubated at RT for 20 minutes and put in $4-\mu \mathrm{L}$ well. The fabricated sensor chip was immersed in the sample solution, and the frequency change $(\mathrm{nm}$, corresponding to binding) was measured for 360 seconds.

\section{Results}

\subsection{Inhibition of Cellular Uptake of Liposomes (LPS) by Myr47}

Since the cellular uptake of HBsAg by HepG2 cells (no NTCP expression) was efficiently inhibited by Myr47 [11], we speculated that this inhibitory activity was not directly related to HBV envelope proteins, but to the lipid nanoparticle structure. In this study, we therefore tried to use LPs, instead of HBsAg and HBV as a starting point for the subsequent analyses.

When DiD (fluorophore)-labeled LPs were incubated with HepG2 cells (no NTCP expression) for 3 hours, efficient cellular uptake of LPs was observed under confocal laser microscopy (Figure 1, left upper panel). Addition of $500 \mathrm{nM}$ Myr47 was found to inhibit 
the cellular uptake of LPs (right upper panel). Flow cytometric analysis also demonstrated that $500 \mathrm{nM}$ Myr47 inhibited nearly $90 \%$ of the cellular uptake of LPs (Figure 2). Together, as previously observed with HBsAg [11], Myr47 can inhibit the cellular uptake of LPs independent of NTCP expression.

Next, we evaluated if $\mathrm{N}$-myristoylation affects the inhibition activity of Myr47 by using N-myristoyl residue-less Myr47 mutant, aa2-48, which cannot inhibit the cellular entry of HBV [14]. While Myr47 inhibited the uptake of LPs, aa2-48 could not inhibit the uptake (Figure 1, left lower panel; Figure 2). This result suggested that N-myristoyl residue is indispensable for the inhibition of cellular uptake of LPs.

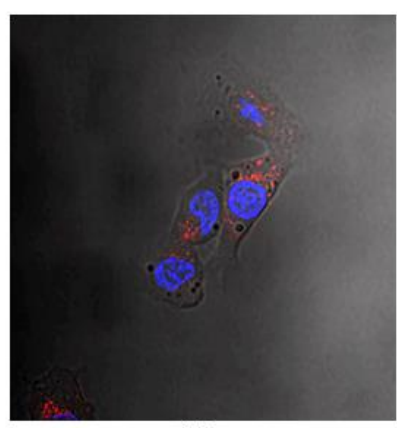

LPS

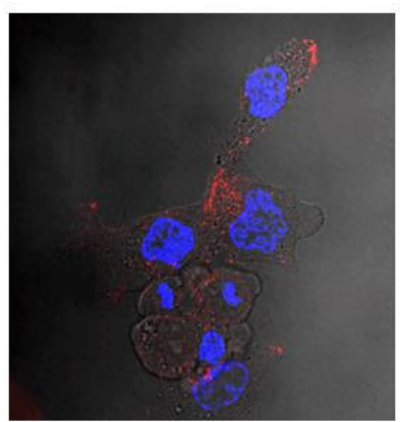

LPs + 500 nM aa2-48

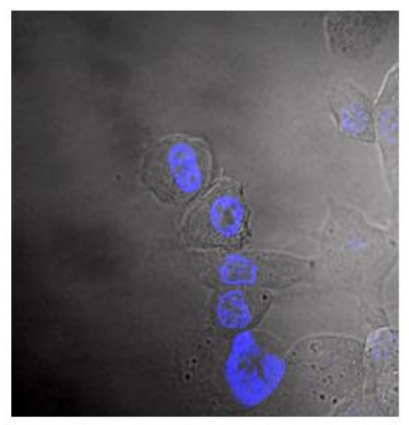

LPs + 500 nM Myr47

Figure 1. Inhibitory effect of Myr47 on the cellular LP uptake by HepG2 cells (no NTCP expression). HepG2 cells were incubated with DiD-LPs $(10 \mu \mathrm{g} / \mathrm{mL})$ in serum-free DMEM containing Myr47 or its mutant $(500 \mathrm{nM})$ for 3 hours at $37^{\circ} \mathrm{C}$, and then observed under confocal laser scanning microscopy. Nucleus (cyan) were stained with Hoechst 33342 (2 $\mu \mathrm{g} / \mathrm{mL}$ ), LPs (red) were stained with DiD.

\subsection{N-Myristoylation-dependent Interaction between Myr47 and LPS}

Considered from the above results, we considered that Myr47 interacts with LPs and thereby interferes the cellular uptake of LPs in an NTCP-independent manner, and therefore examined the interaction of Myr47 with LPs by using an Alphascreen based on Amplified Luminescence Proximity Homogeneous Assay [15]. Both FITC-labeled LPs and biotin-labeled Myr47 were fixed on anti-FITC IgG-conjugated protein A acceptor beads and streptavidin donor beads, respectively. As shown in Figure 3, the interaction of Myr47 with LPs gave strong luminescence, which was comparable level of positive control, while Myr47 mutant aa2-48 gave no luminescence. These results strongly suggested that Myr47 can anchor to the lipid bilayer of LPs with myristoyl residue like other N-myristoylated peptides [16]. 


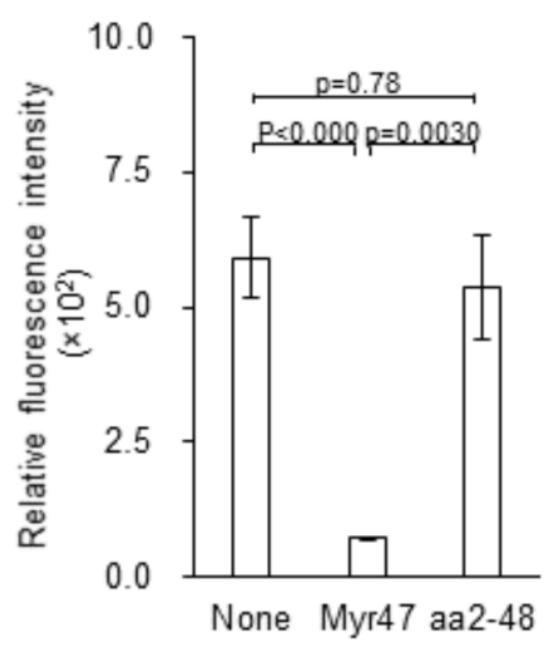

Figure 2. Inhibitory effect of Myr47 on the cellular LP uptake by HepG2 cells (no NTCP expression). HepG2 cells were incubated with DiD-LPs ( $5 \mu \mathrm{g} / \mathrm{mL})$ in serum-free DMEM containing Myr47 or its mutant $(500 \mathrm{nM})$ for 3 hours at $37^{\circ} \mathrm{C}$, and then analyzed with flow cytometry. Data are shown as means \pm S.D. $(\mathrm{N}=3)$.
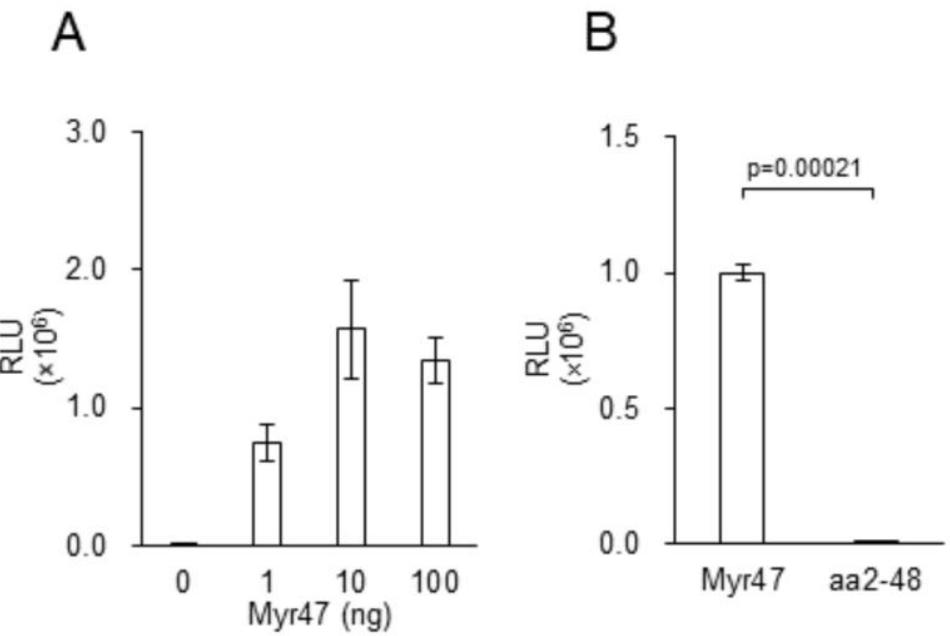

Figure 3. In vitro direct interaction of Myr47 with LPs. (A) Interaction of Myr47 with LPs analyzed by Alphascreen assay. (B) Interaction of Myr47 or aa2-48 with LPs analyzed by Alphascreen assay. Data are shown as means \pm S.D. $(N=3)$.

\subsection{Effect of Amino Acid Sequence on the Myr47-mediated Inhibitory Activity}

Various Myr47 mutants had been examined to inhibit the establishment of HBV infection (mainly, cellular entry of HBV) [14]. In this study, three N-myristoylated Myr47 mutants that lost HBV infection inhibitory activity (i.e., Ala11-15, d-11,13, Scrambled) and one N-myristoyl residue-less Myr47 (i.e., aa2-48) were synthesized (Table 1). Under the same condition of Figure 2, these mutants were examined for the cellular uptake of LPs by using flow cytometric analysis (Figure 4A). All N-myristoylated Myr47 mutants inhibited cellular uptake of LPs, whereas N-myristoyl residue-less Myr47 mutant could not inhibit the cellular uptake. We then analyzed the interaction between these Myr47 mutants and LPs by using Alphascreen as described above (Figure 3). All $\mathrm{N}$-myristoylated Myr47 mutants could interact with LPs efficiently to similar extents of Myr47 (Figure 4B). These results indicated that the inhibitory activity of Myr47-mediated cellular LP uptake requires N-myristoyl residue but neither the Myr47 specific sequence nor NTCP expression. 
Table 1. Myr47 and its mutant peptides.

\begin{tabular}{cc}
\hline Name & Sequence \\
\hline Myr47 & Myr-GTNLSVPNPLGFFPDHQLDPAFGANSNNPDWDFNPNKDQWPEANQVK-biotin \\
aa2-48 & GTNLSVPNPLGFFPDHQLDPAFGANSNNPDWDFNPNKDQWPEANQVK-biotin \\
Ala11-15 & Myr-GTNLSVPNPAAAAADHQLDPAFGANSNNPDWDFNPNKDQWPEANQVK-biotin \\
\hline$d-11,13$ & Myr-GTNLSVPNPLGFFPDHQLDPAFGANSNNPDWDFNPNKDQWPEANQVK-biotin \\
Scrambled (Scr) & Myr-TNNDPFKRTDLAWNGFDSVAQNDLLPNPPFNNWGDGSHQPADAKPFTK-biotin \\
\hline
\end{tabular}

Mutated residues were underlined. $D$-amino acids were marked with asterisks. The amino acid residue composition of Myr47 and Scrambled (Scr) lipopeptide is almost identical.

A

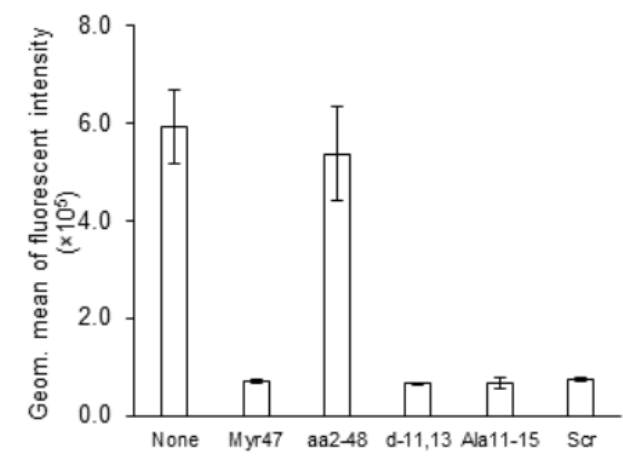

B

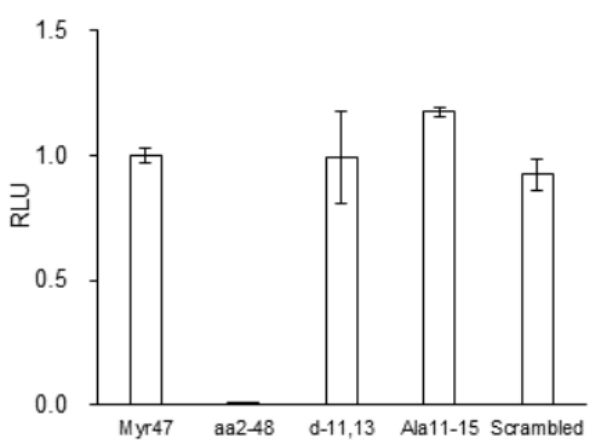

Figure 4. Effect of Myr47 and its mutants on the cellular LP uptake activity and the in vitro direct interaction with LPs. (A) HepG2 cells were incubated with DiD-LPs $(5 \mu \mathrm{g} / \mathrm{mL})$ in serum-free DMEM containing Myr47 or its mutants (500 nM) for 3 hours at $37^{\circ} \mathrm{C}$, and then analyzed with flow cytometry. (B) Interaction of Myr47 or its mutants with LPs analyzed by Alphascreen assay. Data are shown as means \pm S.D. $(\mathrm{N}=3)$.

\subsection{Effect of Myr47 on the ApoE3 (Apolipoprotein E3)-LPs Interaction}

Recently, ApoE3 was reported as an indispensable factor to establish HBV infection, presumably enhancing the cellular entry of HBV by forming ApoE3-HBV complex [12]. For decipherment of the mechanism underlying Myr47-mediated inhibition of cellular LP uptake, HepG2 cells (no NTCP expression) were incubated in serum-free medium (not containing ApoE) and contacted with DiD-labeled LPs for 3 hours in the presence of Myr47 or its mutants with/without $25 \mu \mathrm{g} / \mathrm{mL}$ ApoE3. Cellular uptake of LPs was measured by flow cytometric analysis (Figure 5A). When ApoE3 was added to medium, the cellular uptake of LPs was increased up to 6-fold compared to without the addition of ApoE3. Addition of Myr47 or N-myristoylated mutants completely inhibited the uptake of LPs, while N-myristoyl residue-less mutant (aa2-48) showed no inhibition.

Next, we measured the in vitro direct interaction between LPs and ApoE3 by bio-layer interferometry (BLI) method. ApoE3 was fixed on the protein A sensor chip bridged by anti-ApoE3 IgG. The sensor chip was dipped into LP solution containing Myr47 or its mutants. Myr47 and its mutants except aa2-48 apparently inhibited the interaction between LPs and ApoE3, whereas aa2-48 inhibited to some extent (Figure 5B). These results corroborated that ApoE3 enhances the cellular uptake of LPs by forming ApoE3-LP complex. N-myristoylated peptide, especially $\mathrm{N}$-myristoyl residue, could interfere the formation of ApoE3-LP complex, thereby inhibiting the cellular uptake of LPs. 


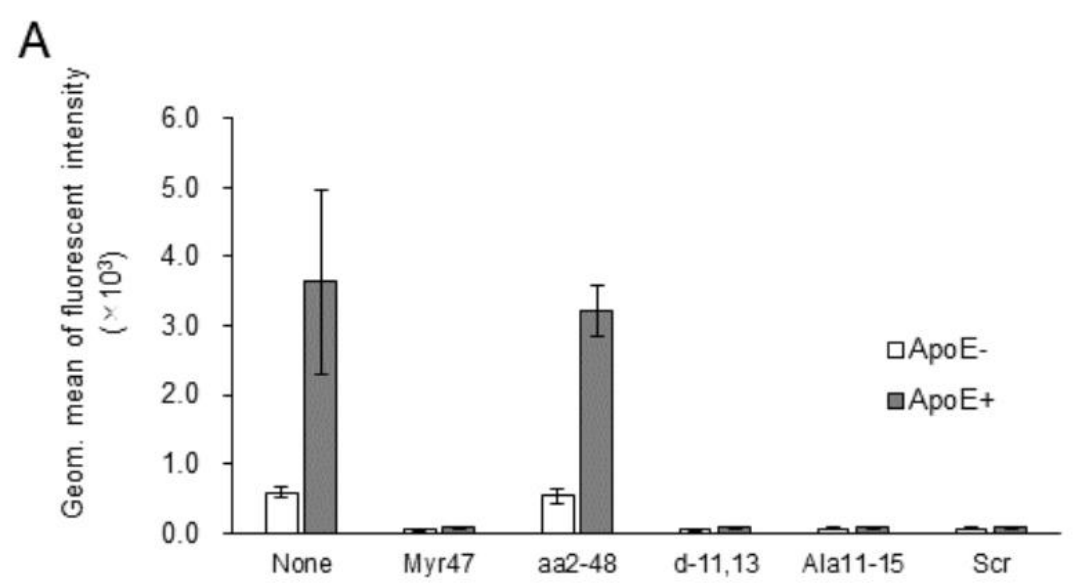

B

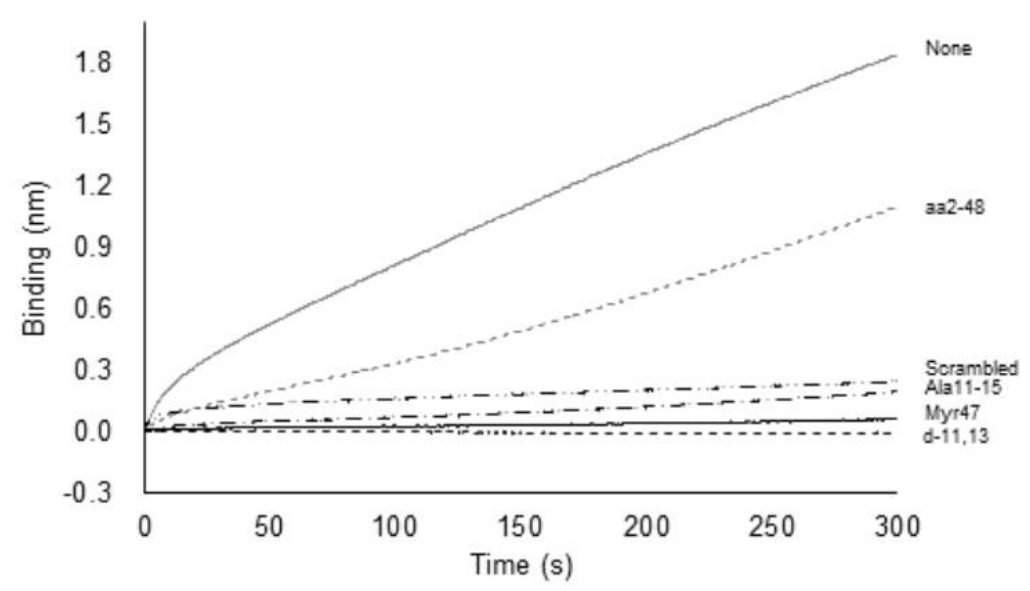

Figure 5. Effect of Myr47 and its mutants on the ApoE3-LP interaction. (A) Effect of ApoE3 on the cellular LP uptake activity in the presence of Myr47 or its mutants. HepG2 cells were incubated with DiD-LPs $(5 \mu \mathrm{g} / \mathrm{mL})$ in serum-free DMEM containing Myr47 or its mutants $(500 \mathrm{nM})$ with/without ApoE3 $(25 \mu \mathrm{g} / \mathrm{mL})$ for 3 hours at $37^{\circ} \mathrm{C}$, and then analyzed with flow cytometry. Data are shown as geometric means \pm S.D. $(\mathrm{N}=3)$. (B) Effect of Myr47 or its mutants on the interaction of LPs and ApoE3 was evaluated with BLItz. Data are shown as means. $(\mathrm{N}=3)$.

\subsection{Effect of Myr47 on the Cellular Uptake of HBsAg}

Myr47 was shown to inhibit the cellular uptake of HBsAg (HBV subviral particle) by HepG2 cells (no NTCP expression) [11]. Herein, we examined if Myr47 mutants affect the cellular uptake of HBsAg by flow cytometric analysis under the same condition (see Figure 4A). Myr47 mutants (except aa2-48) could inhibit almost 70\% of cellular HBsAg uptake, which is comparable level of My47 (Figure 6A). Next, the interaction of Myr47 or its mutants with HBsAg was measured by Alphascreen under the same condition (see Figures 3 and 4B). Both HBsAg and Myr47 were fixed onto anti-S (main constituent of HBsAg) IgG-conjugated protein A acceptor beads and streptavidin donor beads, respectively. Myr47 was found to interact with HBsAg efficiently (Figure 6B). Myr47 mutants (except aa2-48) also interacted with HBsAg in N-myristoyl residue-dependent manner (Figure 6C), as well as LP-Myr47 interaction, suggesting N-myristoyl residue anchors to the lipid bilayer of HBsAg.

We tried to analyze the ApoE3-HBsAg interaction by BLI method, but it was not successful. Because the HBsAg used was derived from HB patient's plasma, it is thought that blood borne ApoE binds to HBsAg, and that this ApoE interferes with the BLI assay. As a corroboration, the cellular uptake inhibition of HBsAg by Myr47 (approximately $70 \%$ inhibition, see Figure 6A) was lower than that of LPs (approximately $90 \%$ inhibition, see Figure 4 A). 


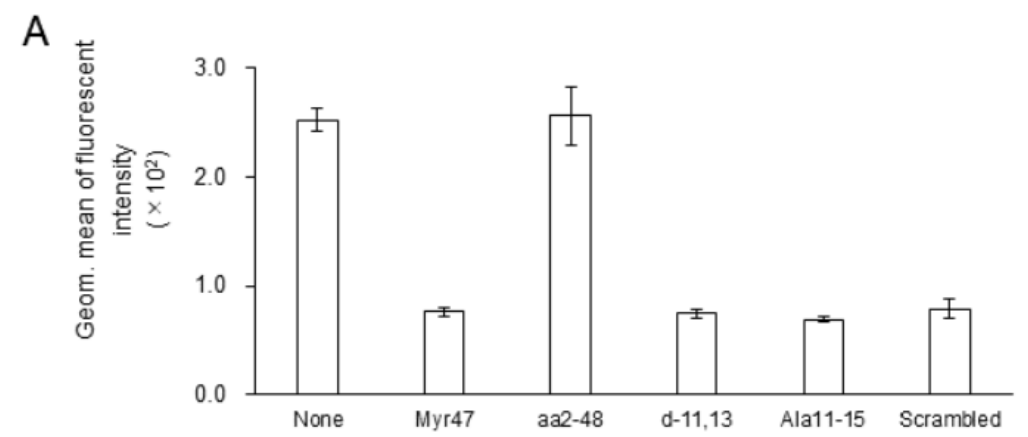

B

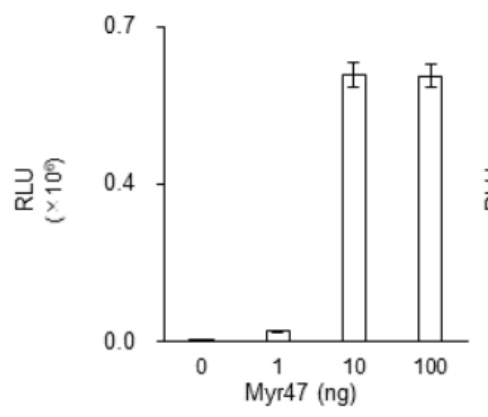

C

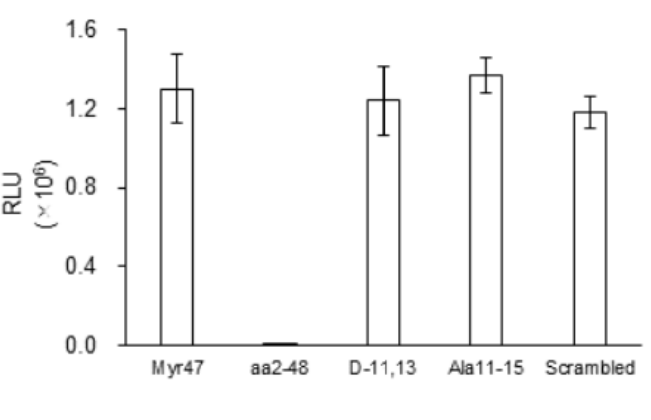

Figure 6. Effect of Myr47 and its mutants on the cellular HBsAg uptake activity and the in vitro direct interaction with HBsAg. (A) HepG2 cells were incubated with CellVue-labeled HBsAg ( $5 \mu \mathrm{g} / \mathrm{mL}$ as protein) in serum-free DMEM containing Myr47 or its mutants $(500 \mathrm{nM})$ for 3 hours at $37^{\circ} \mathrm{C}$, and then analyzed with flow cytometry. (B) Interaction of Myr47 with HBsAg analyzed by Alphascreen assay. (C) Interaction of Myr47 or its mutants with HBsAg analyzed by Alphascreen assay. Data are shown as means. $(\mathrm{N}=3)$.

\section{Discussion}

Our group recently reported that Myr47 inhibits the uptake of HBsAg by HepG2 cells (no NTCP expression) [11]. In this study, flow cytometric analysis revealed that the cellular LP uptake by HepG2 cells was inhibited by Myr47 and its mutants. The inhibitory activity depends on the $\mathrm{N}$-myristoyl residue but not on the amino acid sequence of lipopeptides. Subsequent analysis by Alphascreen revealed that Myr47 and its mutants interact with LPs in an N-myristoyl residue-dependent manner. Meanwhile, ApoE3 enhanced the uptake of LPs by HepG2 cells, which was strongly inhibited by N-myristoyl residue of Myr47 and its mutants. Furthermore, the interaction between ApoE3 and LPs was inhibited by Myr47 and its mutants in an N-myristoyl residue-dependent manner but not in an amino acid sequence-dependent manner.

ApoE3 can interact to the surface of lipid nanoparticles (i.e., LPs, remnant lipoproteins) through a lipid-binding site residing on the C-terminal half and promote the endocytosis of lipid nanoparticles via heparan sulfate proteoglycan (HSPG), low-density lipoprotein receptor (LDLr), or LDLr-related protein (LRP) [13,17]. Since N-myristoylated peptides are spontaneously anchored to lipid bilayers of LPs [18], we herein hypothesized that Myr47 and its mutants anchored on the surface of lipid nanoparticles increased steric hindrance and then caused the inhibition of ApoE3 binding to the surface of nanoparticles. To our knowledge, it is indicated for the first time that fatty acyl chain of lipopeptides contributes to the inhibition of apolipoprotein-mediated endocytosis by modifying the surface of lipid nanoparticles. When therapeutic agent-containing lipid nanoparticles are used as a DDS (drug delivery system) nanocarrier targeting specific organs/cells in vivo, the surface of nanocarriers are usually modified only with targeting molecules (e.g., antibodies, homing peptides, sugar chains). These DDS nanocarriers are unexpectedly trapped by scavenger receptors (i.e., LDLr, LRP) in reticuloendothelial system. Dual surface modification of DDS nanocarriers with targeting molecules and lipopeptides may improve the delivering efficiency to target organs/cells in vivo. 
We examined the effects of Myr47 and its mutants on the cellular uptake of HBV subviral particle (HBsAg; as a mimic of HBV) by HepG2 cells (no NTCP expression). These lipopeptides interacted with the HBsAg surface through their myristoyl residue and inhibited the cellular uptake of HBsAg in an N-myristoyl residue-dependent manner not in an amino acid sequence-dependent manner. HBV (HBsAg as well) was shown to interact with ApoE3 [12]. Hence, we speculated that lipopeptides inhibit the uptake of HBsAg by repelling the access of ApoE3 to HBsAg likewise the case of LPs. However, unlike LPs, the addition of ApoE3 did not enhance the uptake of HBsAg by HepG2 cells (data not shown). Since HBsAg used is derived from patient's plasma and probably had formed complex to some extent with bloodborne ApoE3, the cellular uptake of HBsAg would not be enhanced sufficiently.

In the early stages of HBV infection, HBV initially interacts with cell-surface HSPG, enters the endosomes, interacts with NTCP, sheds its envelope, and release its components into the cytoplasm for establishing HBV infection. It has been widely accepted that Myr47 prevents the establishment of HBV infection by inhibiting the interaction of HBV with NTCP in competitive manner [19], and the inhibitory activity of Myr47 depends on both amino acid sequence and myristoyl residue. Meanwhile in this study, Myr47 and its mutants could inhibit the cellular uptake of HBsAg by HepG2 (no NTCP expression) in a myristoyl residue-dependent manner and not in an amino-acid sequence-dependent manner. These results indicated that the cellular uptake of HBsAg (presumably HBV as well) is independently mediated by two endocytic cascades, NTCP via HSPG and HSPG alone. Since Myr47 effectively inhibits the establishment of HBV infection whereas its mutants not [14], the former endocytic cascade is indispensable for the establishment of $\mathrm{HBV}$ infection, and the later one is not substantially involved in the establishment. For clarifying the significance of two endocytic cascades in the life cycle of HBV, final subcellular destination of each HBV should be determined in future research.

\begin{abstract}
Author Contributions: Conceptualization, M.S. and S.K.; methodology, M.N. and Y.T.C.; writing-original draft preparation, M.N. and M.S.; writing-review and editing, M.N. and S.K.; supervision, S.K.; project administration, S.K.; funding acquisition, S.K. All authors have read and agreed to the published version of the manuscript.

Funding: This work was supported by Japan society for the promotion of science (KAKENHI, Grant-in-Aid for Scientific Research (S) (16H06314, to S.K.)), the Japan Agency for Medical Research and Development (17cm0106214h0002, 17fk0310105h0001, to S.K.), and "Dynamic Alliance for Open Innovation Bridging Human, Environment and Materials", of the Ministry of Education, Culture, Sports, Science and Technology of Japan (MEXT, to S.K.).
\end{abstract}

Conflicts of Interest: The authors declare no conflict of interest.

\title{
References
}

1. Neuveut, C.; Wei, Y.; Buendia, M.A. Mechanisms of HBV-related hepatocarcinogenesis. J. Hepatol. 2010, 52, 594-604.

2. Heermann, K.H.; Goldmann, U.; Schwartz, W.; Seyffarth, T.; Baumgarten, H.; et al. Large surface proteins of hepatitis B virus containing the pre-s sequence. J. Virol. 1984, 52, 396-402.

3. Neurath, A.R.; Kent, S.B.H.; Strick, N.; Parker, K. Identification and chemical synthesis of a host cell receptor binding site on hepatitis B virus. Cell 1986, 46, 429-436.

4. Pontisso, P.; Ruvoletto, M.G.; Gerlich, W.H.; Heermann, K.H.; Bardini, R.; et al. Identification of an attachment site for human liver plasma membranes on hepatitis B virus particles. Virology 1989, 173, 522-530.

5. Klingmüller, U.; Schaller, H. Hepadnavirus infection requires interaction between the viral pre-S domain and a specific hepatocellular receptor. J. Virol. 1993, 67, 7414-7422.

6. Seyec, J.L.; Chouteau, P.; Cannie, I.; Guguen-Guillouzo, C.; Gripon, P. Infection process of the hepatitis B virus depends on the presence of a defined sequence in the pre-S1 domain. J. Virol. 1999, 73, 2052-2057.

7. Gripon, P.; Le seyec, J.; Rumin, S.; Guguen-guillouzo, C. Myristylation of the hepatitis B virus large surface protein is essential for viral infectivity. Virology 1995, 213, 292-299.

8. Bruss, V.; Hagelstein, J.; Gerhardt, E.; Galle, P.R. Myristylation of the large surface protein is required for hepatitis B virus in vitro infectivity. Virology 1996, 218, 396-399.

9. Glebe, D.; Urban, S.; Knoop, E.V.; Çağ, N.; Krass, P.; et al. Mapping of the hepatitis B virus attachment site by use of infection-inhibiting preS1 lipopeptides and Tupaia hepatocytes. Gastroenterology 2005, 129, 234-245. 
10. Yan, H.; Zhong, G.; Xu, G.; He, W.; Jing, Z.; et al. Sodium taurocholate cotransporting polypeptide is a functional receptor for human hepatitis B and D virus. eLife 2012, 1, e00049.

11. Somiya, M.; Liu, Q.; Yoshimoto, N.; Iijima, M.; Tatematsu, K.; et al. Cellular uptake of hepatitis B virus envelope L particles is independent of sodium taurocholate cotransporting polypeptide, but dependent on heparan sulfate proteoglycan. Virology 2016, 497, 23-32.

12. Qiao, L.; Luo, G.G. Human apolipoprotein E promotes hepatitis B virus infection and production. PLoS Pathog. 2019, 15, e1007874.

13. Yan, X.; Kuipers, F.; Havekes, L.M.; Havinga, R.; Dontje, B.; et al. The role of apolipoprotein E in the elimination of liposomes from blood by hepatocytes in the mouse. Biochem. Biophys. Res. Commun. 2005, 328, 57-62.

14. Schulze, A.; Schieck, A., Ni, Y., Mier, W., Urban, S. Fine mapping of pre-S sequence requirements for hepatitis B virus large envelope protein-mediated receptor interaction. J. Virol. 2010, 84, 1989-2000.

15. Seethala, R.; Prabhavathi, F. Homogeneous Assays: AlphaScreen. In Handbook of drug screening.; Marcel Dekker: New York, U.S.A., 2001; pp. 106-110.

16. Johnson, D.R.; Bhatnagar, R.S.; Knoll, L.J.; Gordon, J.I. Genetic and biochemical studies of protein N-myristoylation. Ann. Rev. Biochem. 1994, 63, 869-914.

17. Mahley, R.W., Ji, Z.S. Remnant lipoprotein metabolism: key pathways involving cell-surface heparan sulfate proteoglycans and apolipoprotein E. J. Lipid Res. 1999, 40, 1-16.

18. Murray, D.; Ben-Tal, N.; Honig, B.; McLaughlin, S. Electrostatic interaction of myristoylated proteins with membranes: simple physics, complicated biology. Structure 1997, 5, 985-989.

19. Zhang, Z.; Zehnder, B.; Damrau, C.; Urban ,S. Visualization of hepatitis B virus entry - novel tools and approaches to directly follow virus entry into hepatocytes. FEBS Lett. 2016, 590, 1915-1926. 